

\title{
Improved unfolding by detrending of statistical fluctuations in quantum spectra
}

\author{
I.O. Morales, E. Landa, P. Stransky, A. Frank
}

\section{To cite this version:}

I.O. Morales, E. Landa, P. Stransky, A. Frank. Improved unfolding by detrending of statistical fluctuations in quantum spectra. Physical Review E: Statistical, Nonlinear, and Soft Matter Physics, 2011, 84, pp.016203. 10.1103/PhysRevE.84.016203 . in2p3-00601797

\section{HAL Id: in2p3-00601797 https://hal.in2p3.fr/in2p3-00601797}

Submitted on 20 Jun 2011

HAL is a multi-disciplinary open access archive for the deposit and dissemination of scientific research documents, whether they are published or not. The documents may come from teaching and research institutions in France or abroad, or from public or private research centers.
L'archive ouverte pluridisciplinaire HAL, est destinée au dépôt et à la diffusion de documents scientifiques de niveau recherche, publiés ou non, émanant des établissements d'enseignement et de recherche français ou étrangers, des laboratoires publics ou privés. 


\title{
An improved unfolding by detrending of statistical fluctuations in quantum spectra
}

\author{
Irving O. Morales, ${ }^{1}$ E. Landa, ${ }^{2}$ P. Stránský, ${ }^{2,3}$ and A. Frank ${ }^{2}$ \\ ${ }^{1}$ Grand Accélérateur National d'Ions Lourds, GANIL, CEA/DSM - CNRS/IN2P3, \\ Bd. Henri Becquerel, BP 55027, F-140\%6, Caen Cedex 5, France \\ ${ }^{2}$ Instituto de Ciencias Nucleares, Universidad Nacional Autónoma de México, \\ Apartado Postal 70-543, 04510 México, D.F., Mexico \\ ${ }^{3}$ Institute of Particle and Nuclear Physics, Faculty of Mathematics and Physics, \\ Charles University, V Holešovičkách 2, 18000 Prague, Czech Republic
}

(Dated: June 6, 2011)

\begin{abstract}
A fundamental relation exists between the statistical properties of the fluctuations of the energy level spectrum of a Hamiltonian and the chaotic properties of the physical system it describes. This relationship has been addressed previously as a signature of chaos in quantum dynamical systems. In order to properly analyze these fluctuations, however, it is necessary to separate them from the general tendency, namely, its secular part. Unfortunately this process, called unfolding, is not trivial and can lead to erroneous conclusions about the chaoticity of a system. In this paper we propose a technique to improve the unfolding procedure for the purpose of minimizing the dependence on the particular procedure. This technique is based on detrending the fluctuations of the unfolded spectra through the empirical mode decomposition method.
\end{abstract}

PACS numbers: 05.40.-a, 05.45.-a, 05.45.Tp

\section{INTRODUCTION}

The relation between classical dynamical systems and their quantum counterparts is an important aspect of contemporary research. Of particular relevance is the study of the so called quantum chaos, the relation between classical chaos and quantum mechanical systems. One of the fundamental signatures of quantum chaos is the conjectured link between the statistical fluctuations of the energy spectrum and the integrability or chaotic properties of the Hamiltonian [1-3]. This relation has been studied in a large diversity of systems, including microwave cavities, atoms, molecules and nuclei. This kind of spectral analysis has become one of the main tools for the study of quantum chaos, whose fundamental objective is to characterize the properties of these fluctuations [4] To tackle this problem it is assumed that the density of states $\rho(E)$ can be separated into a secular and a fluctuating part

$$
\rho(E)=\overline{\rho(E)}+\widetilde{\rho(E)} .
$$

While the secular part $\overline{\rho(E)}$ usually indicates particular characteristics of the system under consideration, the fluctuating part reflects universal properties of physical systems. However, to separate these two components of the spectral density is a major problem. The procedure used to extract the information of the fluctuating part in the spectra is called unfolding. In essence, this procedure consists in mapping the spectrum of the excited states $E_{i}$ into a dimensionless spectrum $\epsilon_{i}$, whose main characteristic is a mean level density normalized to 1 . It has been widely appreciated that this process is not trivial [4], but the unfolding details are usually omitted in the literature. Of special importance to the present work is the analysis reported on [5], which demonstrates that the statistics that measures long-range correlations strongly depends on the unfolding procedure and in the end, this dependence can give rise to misleading results in regard to the chaoticity of quantum systems. The main goal of the present paper is to propose an unfolding technique which leads to reliable signatures of chaos. This is achieved subtracting the residual trend which remains after the initial unfolding process. This is achieved by means of the empirical mode decomposition technique.

\section{UNFOLDING PROCEDURE}

Given a spectral density $\rho$ it is possible to define an accumulated level density $N(E)$, which measures the number of levels up to a certain energy $E$ in the system

$$
N(E)=\int_{-\infty}^{E} d E^{\prime} \rho\left(E^{\prime}\right) .
$$

In this way we can distinguish between a smooth and a fluctuating part

$$
N(E)=\overline{N(E)}+\widetilde{N(E)}
$$

The unfolding procedures usually involves an estimation of a functional shape for the smooth part of the spectral density $\overline{\rho(E)}$ or for the smooth part of the accumulated level density $\overline{N(E)}$. Once the estimated accumulated density is known a new dimensionless spectrum $\epsilon_{i}$ is obtained, where the levels are given by 


$$
\epsilon_{i}=\overline{N\left(E_{i}\right)}
$$

The problem is how to find an appropriate smooth estimation for the secular part of the level density. When possible, the spectral density is obtained theoretically using the knowledge of the low order energy moments, which are related to the trace of powers of the hamiltonian matrix [6]. If this is not possible, then it is necessary to obtain it from the spectrum itself. Several procedures have been proposed in order to unfold this kind of spectra. The easiest unfolding approach is to assume a polynomial dependence for the accumulated level density [7]. In this approach a high order polynomial interpolation for $N(E)$ is taken, the only parameter being the order of the polynomial approximation. However, this estimation can be quite inaccurate, since the polynomial function may have no relation with the physical properties of the system. It is also possible to consider a linear fit of $N(E)$ on an interval containing $\nu$ levels around the level $E_{i}$, and a moving average then taken through the whole spectrum. The $\nu$ value is used as a free parameter [8]. More sophisticated approaches for the unfolding involve Fourier broadening of the step functions, which reflect the accumulated density or a Gaussian broadening of the level density. While usually this density is conformed by a summation of delta functions for every level, under this approach the delta functions are substituted by an average gaussian level density. It is also possible to include local effects in this procedure [9].

Regardless of which method is used to unfold the fluctuating part of the spectra and even if a good estimation for the accumulated level density is obtained, this approximation may not be good enough. If this is the case the unfolding does not capture the full behavior of the smooth part of the spectrum. The net effect is that spurious long-range correlations are introduced. These spurious correlations are responsible for the misleading signatures of quantum chaos that may occur [5]. The technique that we propose here does not depend on apriori selection of unfolding functions and, as we shall show, significantly improves the estimation for the smooth part. Throughout this work we carry out an initial unfolding with polynomial approximations as an example, but the results do not depend on this choice and it is possible to improve the estimation starting with any of the previously mentioned procedures.

Once a dimensionless spectrum is obtained, we can calculate the nearest neighbor level spacings defined by

$$
s_{i}=\epsilon_{i+1}-\epsilon_{i}, i=1, \ldots, N-1 .
$$

Recently, a proposal was made to study the fluctuating part of a quantum spectrum as a time series, which gives rise to an alternative signature of quantum chaos [10]. This approach characterizes the spectral fluctuations with the $\delta_{n}$ statistic [11] defined by

$$
\delta_{n}=\sum_{i=1}^{n}\left(s_{i}-\langle s\rangle\right) .
$$

The discrete function $\delta_{n}$ measures the deviations between the energy of the $(n+1)$-th unfolded state and the corresponding energy of an equally spaced energy sequence. The main objective of the analysis is to propose a correspondence between the $\delta_{n}$ statistic and time series, by equating time to the index $n$, which represents the ordering number of the energy levels. It is then possible to apply the usual techniques of time series analysis.

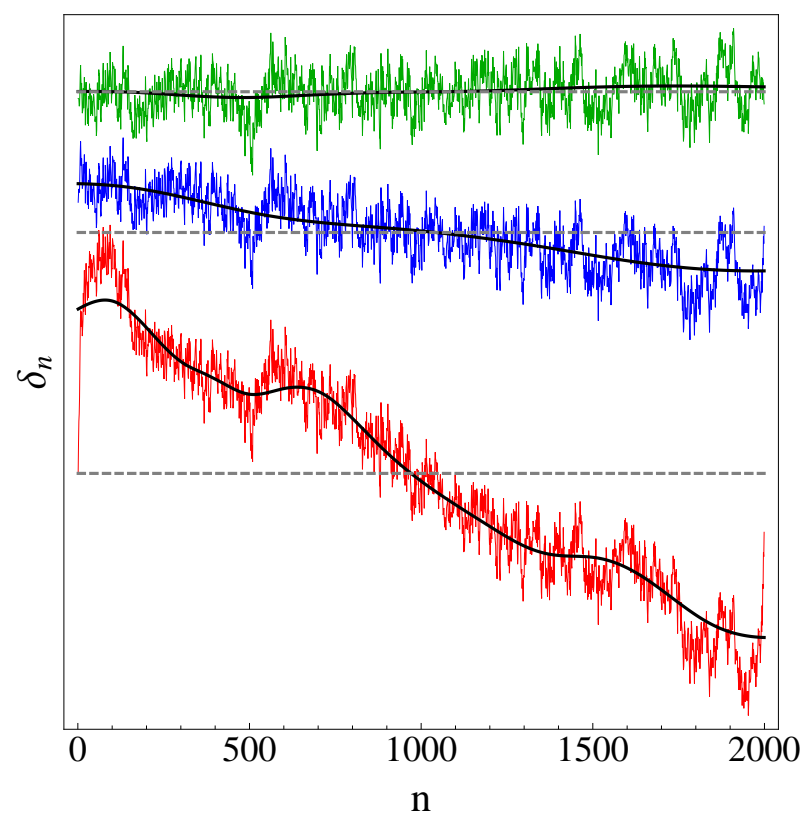

FIG. 1: Color online. $\delta_{n}$ statistic for a GOE spectrum unfolded with polynomials of order 5 (bottom, red online), 9 (middle, blue online) and 13 (top, green online). The dashed lines show the mean on each case and the black solid line shows the trend obtained trough the EMD. Each plot was moved along the vertical axis to avoid overlapping.

As we mentioned above, the result of a defective unfolding is the introduction of spurious correlations in the spectrum fluctuations. In a properly unfolded spectrum all traces of the secular component of the signal should in principle be removed. In terms of time series, this correlations are represented as global trends on the $\delta_{n}$ statistic, i.e. a smoothly varying behavior is present. If the energy spectrum is correctly unfolded then the $\delta_{n}$ should consist only of fluctuations around its constant mean value. Figure 1 shows the effect of 
unfolding with polynomials of different order on the same energy spectrum. The spectrum was obtained from a gaussian orthogonal ensemble (GOE) matrix, a system which is regarded as a paradigm for the study of quantum chaos [3]. The energy spectrum was unfolded with three different polynomials and the resulting $\delta_{n}$ for each case is shown in figure 1 . The bottom (red online) signal shows a $\delta_{n}$ obtained with a fifth order fit, the middle (blue online) one shows the one obtained with a ninth order fit and the top (green online) signal shows the $\delta_{n}$ obtained for a fit with a polynomial of order 13. The plots were displaced along the vertical axis to avoid overlapping. The dashed lines show the mean for each case. If the unfolding procedure were correct, the time series should fluctuate around this mean, but a clear global trend is still present. This trend is shown as a black solid line on each case. In order to solve the problem of an incorrect unfolding we propose to detrend the $\delta_{n}$ time series using the EMD technique.

\section{TRENDS AND EMPIRICAL MODE DECOMPOSITION}

The trend is a basic concept in a large amount of subjects in physics, economics, statistics, sociology, etc. The concept is used specifically in the analysis of data sets. While an intuitive idea of the trend can be used, there is no precise definition of it. In most of the data analysis applications, the trend dominates the global behavior of the data. If a more precise analysis of the phenomena involved is required, then the trend must be substracted in order to reveal the relevant details. Unfortunately, a general algorithm to detrend a time series does not exist, especially in the case where the time series is nonlinear and nonstationary. As in the case of the unfolding procedure, an accurate and detailed description of the detrending procedure is usually avoided in the literature. Recently an attempt to mathematically define the trend has been proposed [12], where the authors put special emphasis on the fact that the trend is part of the data, an intrinsic property of it. Therefore, in order to extract the trend an adaptive method is required, namely, the trend must be derived directly from the data. The empirical mode decomposition technique [13] is especially suited to accomplish a detrending process fully based on the data.

The empirical mode decomposition (EMD) proposes the expansion and decomposition of the data in terms of intrinsic mode functions (IMF) which are derived from the data and conforms an adaptive local and nonlinear basis. This basis is constructed using the physical time scales that characterize the oscillations of the phenomena. The EMD assumes that the time series under analysis is composed of the superposition of several nonstationary processes that take place in different time scales.
The essence of the method is to identify the oscillation modes of the data in a characteristic time scale and then decompose the data into these modes of oscillation. The local time scale of an oscillation is identified by the time lapse between the successive alternations of local maxima and minima. The time series is composed of several oscillations mounted on oscillations corresponding to longer time scales, which in turn are also riding bigger oscillations, and so on. The process of sifting, described below, has been designed in order to untangle these oscillations and extract them from the time series. The oscillating functions in which the time series is decomposed are called intrinsic mode functions. These intrinsic mode functions are defined as functions in which a) the number of extrema and the number of zero crossings are the same or differ at most by one, and b) at any point the mean value of the envelope defined by the local maxima and the envelope defined by the local minima is zero. In general the data oscillations involve many time scales and it is necessary to decompose the time series in its IMFs. The process of sifting to decompose the data into IMFs is as follows:

1. Localize all the local maxima in the time series and connect them with a cubic spline, which defines the upper envelope. Repeat the procedure with the local minima defining the lower envelope.

2. Calculate the mean value of the upper and lower envelopes $m^{(1)}$, as well as the difference with the data points

$$
x_{j}-m_{j}^{(1)}=h_{j}^{(1)}
$$

Since the envelopes include all the extrema, the oscillation between them, $h^{(1)}$, is the oscillation of the data with the finest time scale. Ideally this oscillation should be an IMF, but in practice the process produces new extrema and more iterations are needed to extract an IMF. In this new iterations the obtained component is used as the new data set.

3. Repeat steps 1 and 2 using the newly obtained component as data

$$
h_{j}^{(1)}-m_{j}^{(1,1)}=h_{j}^{(1,1)}
$$

Stop when the last component is an IMF, i.e., when all local maxima are positive and all local minima are negative, and the mean of the envelopes is locally zero, which ensures that the wave is symmetric. The IMF is obtained after $k$ iterations

$$
h_{j}^{(1, k-1)}-m_{j}^{(1, k)}=h_{j}^{(1, k)} .
$$


The function $h^{(1, k)}$ is then designated as an intrinsic mode function of the data and as a component of our new basis

$$
c^{(1)}=h^{(1, k)}
$$

4. Subtract the obtained IMF from the original data

$$
x_{j}-c_{j}^{(1)}=r_{j}^{(1)} .
$$

The residue $r^{(1)}$ is a new version of the data where the finest oscillations have been removed. However, it still contains information of longer period components, so it is necessary to remove these additional components.

5. Repeat steps 1 to 4 , in order to extract all the IMF components in the original data, using as new data on each iteration the residue obtained in the previous one

$$
r^{(1)}-c^{(2)}=r^{(2)}, \ldots, r^{(m-1)}-c^{(m)}=r^{(m)},
$$

where $\mathrm{m}$ is the number of IMFs in the data. This process can be stopped when the $m$ th residue is a monotonic function and no more IMFs can be extracted. The last residue is called the trend of the data. It is important to notice that any residue constitutes a trend for the previously extracted oscillation, i.e., $r^{(i)}$ is the trend followed by the $c^{(i)}$ intrinsic mode function. For a discussion about the specific details of the algorithm and details about its convergence see [13-15].

After following this procedure, it is possible to express the original data in terms of the IMFs as

$$
x_{j}=\sum_{i=1}^{m} c^{(i)}+r^{(m)} .
$$

We are interested in the last residue $r^{(m)}$, which is the global trend.

The trend should be a constant for a $\delta_{n}$ obtained from a correctly unfolded spectrum. Figure 1 shows as solid black lines the trends obtained through the EMD for the same spectrum, previously unfolded with polynomials of order 5,9 and 13. Two undesired effects are apparent in this plot, the first one related to the global trend, which is still visible after the unfolding procedure. In each case it is evident that the EMD technique captures the global behavior, so it is possible to subtract it. The second effect is more subtle and has to do with the fact that the values for the $\delta_{n}$ on the edges of the spectrum change abruptly, especially when the order of the original polynomial fit is low. If this is the case, the EMD decomposition is not well suited for these areas, due to the border conditions of the cubic spline interpolations. In order to avoid this effect we drop $5 \%$ of the eigenvalues at the beginning and at the end of the spectrum.

In order to decrease the dependence on the unfolding procedure, we propose to construct a new detrended fluctuation, subtracting the trend obtained with the EMD, $\delta_{n}^{\prime}=\delta_{n}-r^{(m)}$. Figure 2 shows the result of excluding $5 \%$ of the eigenvalues on the edges of the spectrum and subtracting the residual trend from the original $\delta_{n}$ statistic (Fig.1). It is remarkable how in a qualitative examination the main features of the fluctuations are present on each plot and apparently display the same features. A subtle difference is appreciated at the last part of the $\delta_{n}^{\prime}$ obtained with a fifth order polynomial. This plot has an extra oscillation around $n=1200$ which is present due to the effect mentioned before, the abrupt change of $\delta_{n}$ values on the edges of the spectrum. In this case dropping $5 \%$ of the eigenvalues may not be enough. However this case is useful to appreciate how the affected part is restricted to the end of the $\delta_{n}^{\prime}$ statistic, i.e., it is a local phenomenon which can be removed. To quantitatively show that the new $\delta_{n}^{\prime}$ statistics are independent of the unfolding is the main goal of the next section.

\section{MEASUREMENTS OF QUANTUM CHAOS}

In order to demonstrate that the $\delta_{n}^{\prime}$ statistic, obtained subtracting the EMD trend, leads to chaos measurements independent of the unfolding procedure, we performed a numerical experiment. We constructed and diagonalized 100 GOE matrices with 2000 eigenvalues per matrix. Then the $\delta_{n}$ and $\delta_{n}^{\prime}$ statistics were computed for each spectrum. Given these two statistics the ensemble behavior of the usual measurements of quantum chaos is compared for different orders of the polynomial fit. The GOE system was chosen because it is a well known example of a system presenting quantum chaos and, in addition, the theoretical result for the usual measurements of chaos are known $[3,10,11]$.

As a first test we choose a quantum chaos measure which is computed using the $\delta_{n}$ statistic, the power law behavior of the power spectrum of the fluctuation time series [10]. The power spectrum of a given time series, $x_{n}$, is defined as

$$
S(k)=\left|\hat{X}_{k}\right|^{2}=\frac{1}{\sqrt{N}} \sum_{n} x_{n} \exp \left(\frac{-2 \pi i k n}{N}\right),
$$

where $\hat{X}_{k}$ is the Fourier transform of $x_{n}$ and $N$ is the 


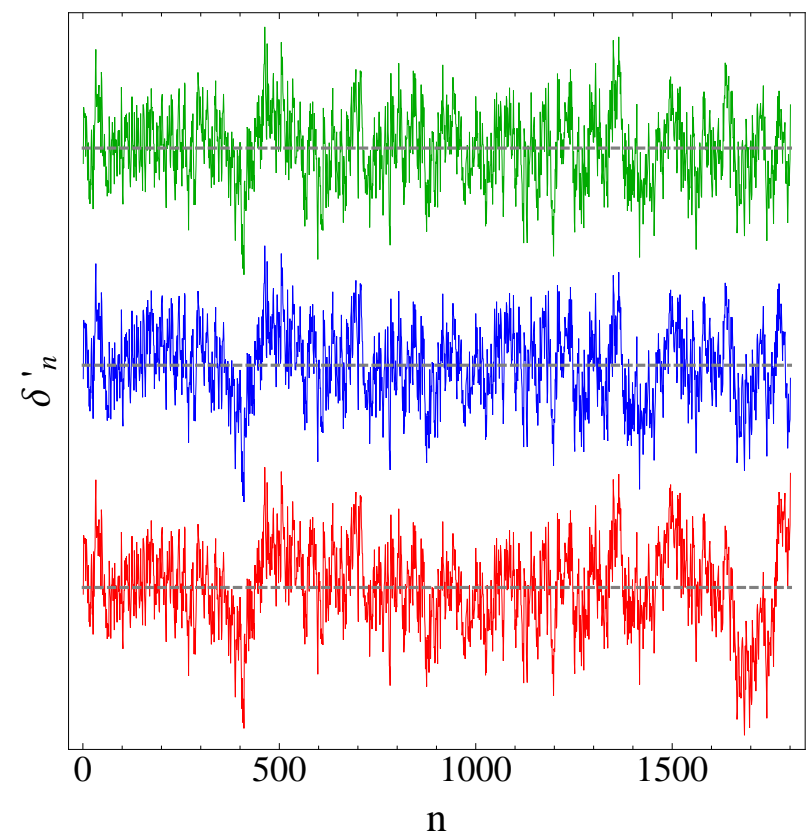

FIG. 2: Color online. Detrended $\delta_{n}$ statistic for a GOE spectrum, definied as $\delta_{n}^{\prime}=\delta_{n}-r^{(m)}$ where $r^{(m)}$ is the trend obtained through EMD. The spectrum was unfolded with polynomials of order 5 (bottom, red online), 9 (middle, blue online) and 13 (top, green online). The dashed lines show the mean on each case. Each plot was moved along the vertical axis to avoid overlapping.

the number of elements in the series. It has been demonstrated that the power spectrum of the fluctuations of quantum spectra follows a power law dependence $\langle S(k)\rangle \sim 1 / k^{\beta}$, this relation is only an approximation, valid without taking into account the highest frequencies of the spectrum. Therefore in the numerical analysis, we take only the region of frequencies which follows a power law behavior, [10, 16-18]. In the case of an integrable system the value of the exponent approaches $\beta=2$, because the inter-level spacings behave as random independent variables [2]. For the GOE system the value $\beta=1$ corresponds to the fact that correlations exists on all available scales. According to this, the power spectrum of a correctly unfolded GOE spectrum must follow the power law $\langle S(k)\rangle \sim 1 / k$. The numerical experiment compares the effectiveness of detecting the presence of quantum chaos using both statistics, the $\delta_{n}$ fluctuation obtained by the traditional unfolding of the spectrum and the one without trend, the $\delta_{n}^{\prime}$. In order to compare the ensemble behavior of the system, an ensemble average power spectrum is computed using the 100 individual power spectra, which lowers dispersion and brings out the power law behavior of the system.

The results of the numerical experiment are compared in figure 3. This figure shows the values of the exponent

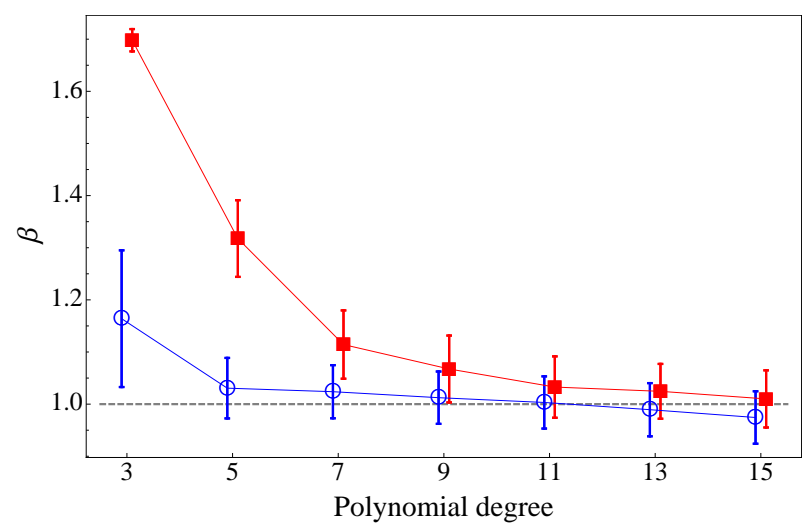

FIG. 3: Color online. Value of the $\beta$ exponent of the power law behavior in the average power spectrum of the fluctuations as a function of the degree of the polynomial fit used to unfold the quantum spectra. Two cases are shown, the $\beta$ exponent values for the $\delta_{n}$ fluctuation (filled squares, red online) and the case for the $\delta_{n}^{\prime}$ fluctuation obtained detrending the previous one (empty circles, blue online). The dashed line shows the theoretical result for the GOE. The error bars show the standard deviation of the ensemble.

$\beta$ obtained for different orders of the unfolding polynomial fit. The filled squares (red online) corresponds to the case of the average power spectrum of the $\delta_{n}$ fluctuation and the empty circles (blue online) shows the case of the power spectrum of the detrended fluctuation $\delta_{n}^{\prime}$. The error bars show the standard deviation using individual fits to the power spectra of each member of the ensemble. The gray dashed line indicates the theoretical value of the $\beta$ exponent for the GOE system. It is evident that the value of $\beta$ depends on the order of the polynomial used to unfold the quantum spectra. If the order of the polynomial is too low then spurious longrange correlations are introduced and the low frequency part of the power spectrum is enhanced producing power law exponents larger than $\beta=1$. However, the use of the EMD to remove the residual trend eliminates these spurious correlations and improves the value of the exponent which gets closer to the theoretical value. When the trend is removed, for all the degrees of the polynomial fit lower than 15 , the $\beta$ value agrees with the theoretical value, except for the example corresponding to the third order polynomial, which is an extreme case.

As the order of the polynomial increases the $\beta$ value approaches the theoretical one. If the order of the polynomial is big enough then the polynomial unfolding destroys correlations and the $\beta$ value tends towards values lower than $\beta=1$. This situation cannot be improved by the use of the EMD because important correlations are missing and cannot be recovered.

Using the detrended fluctuation $\delta_{n}^{\prime}$ it is possible to ob- 
tain a detrended dimensionless spectrum $\epsilon_{i}^{\prime}$ as

$$
\epsilon_{i+1}^{\prime}=\delta_{i}^{\prime}+\epsilon_{1}+i\langle s\rangle,
$$

where $\delta_{n}^{\prime}$ are the fluctuations or the difference between the dimensionless quantum spectrum of the system $\epsilon^{\prime}$ and a regular version of it, $\epsilon_{1}+n\langle s\rangle$. Given this detrended spectrum it is possible to explore the dependence of other measurements of chaos as a function of the order of the polynomial used in the unfolding. The short-range correlations are usually analyzed by means of the nearest neighbor spacing distribution $P(s)$. It is known that the spectra from GOE follow the Wigner surmise. This distribution is not sensitive to the unfolding details [5] and we only mention here that the Wigner distribution is conserved after removing the trend of the fluctuations.

The study of the long-range correlations in quantum spectra is traditionally done by means of the rigidity of the spectrum, which is represented by the $\Delta_{3}$ statistic. The $\Delta_{3}(L)$ is defined as the average over $\gamma$ of the function:

$$
\Delta_{3}(L ; \gamma)=\frac{1}{L} \min _{a, b} \int_{\gamma}^{\gamma+L}[N(E)-a E-b] \mathrm{d} E,
$$

where $N(E)$ is the integrated level density of the unfolded spectrum and $a$ and $b$ are parameters of a linear model which fits $N(E)$ in the energy range $(\gamma, \gamma+L)$. Theoretically the values of the $\Delta_{3}(L)$ for the integrable system and for the GOE are well known [19], in the case of $\operatorname{GOE} \Delta_{3}(L)=(\log (L)-0.0687) / \pi^{2}$ while in the case of an integrable system $\Delta_{3}(L)=1 / 15$.

It is possible to compute the $\Delta_{3}$ statistic for the detrended spectrum and compare it with the $\Delta_{3}$ obtained from the traditionally-unfolded spectrum. Figure 4 shows this comparison. The upper part shows the ensemble average of the $\Delta_{3}$ obtained for the unfolded spectrum using different degrees for the polynomial fit. The dashed gray line shows the predicted theoretical behavior for GOE. We observe that for all polynomial degrees the expected result is followed up to $L=200$, except for the third order polynomial which shows some small deviations. For greater values of $L$ the low order polynomial spectra deviate systematically from the theoretical behavior due to the spurious correlations, which correspond to the residual trend. Since the trend can be represented as low frequency oscillations it is expected that the effect will be reflected in the long-range correlations region. The high order polynomial spectrum also deviates because of the effect mentioned before for the case of the Fourier spectrum analysis, i.e., the polynomial unfolding destroys important correlations. The lower part shows the ensemble average of the $\Delta_{3}$ computed using the detrended spectra from eq (15). The

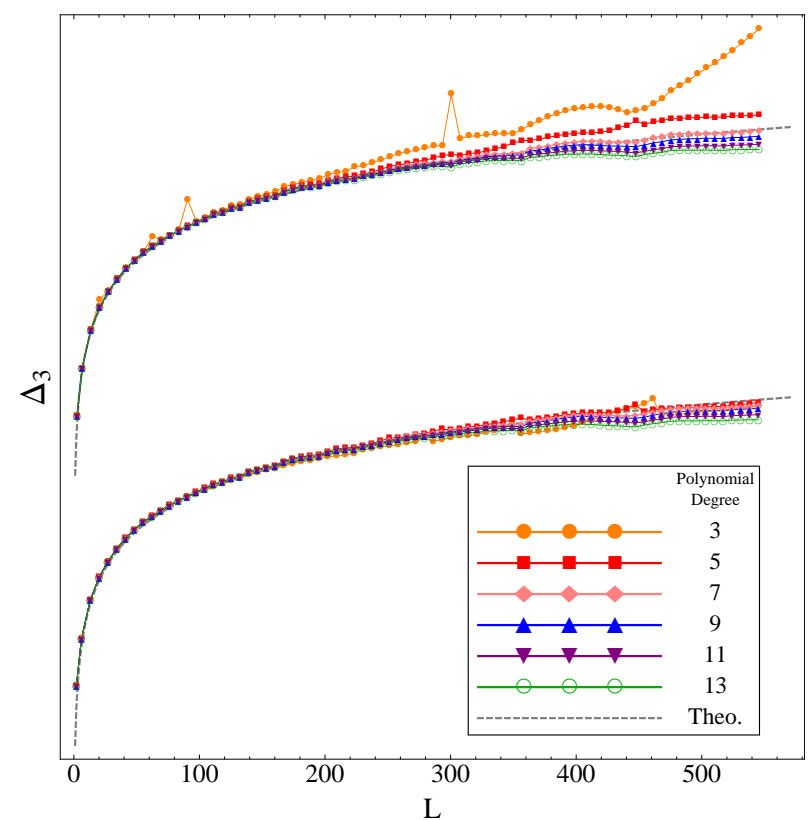

FIG. 4: Color online. Value of the $\Delta_{3}(L)$ averaged over the ensemble. The upper part shows the $\Delta_{3}$ computed using polynomials of different order to unfold the spectra, where the plots have been displaced to avoid overlap. The lower part shows the $\Delta_{3}$ calculated from detrended spectra obtained from the detrended fluctuation $\delta_{n}^{\prime}$. Plots for different degrees of the polynomial fit used to unfold the quantum spectra are shown in both cases. The dashed line shows the theoretical result for the GOE.

effect of subtracting the residual trend of the fluctuations is evident. In the region of small $L$ the deviations for the third order polynomial are gone while in the region of larger $L$ the low order polynomial behavior improves and follows the expected theoretical behavior. The case for the high order polynomials does not improve, because once important correlations are destroyed they cannot be recovered.

The $\Delta_{3}$ statistic is a good measure of the long-range correlations of a quantum spectrum, but it is difficult to obtain a quantitative deviation from the theoretical behavior, as done in the previous example of the Fourier power spectrum by means of the value of the exponent $\beta$. Recently another measurement of the chaoticity of a quantum system has been proposed [20]. This method uses a detrended fluctuation analysis technique [21] (DFA) which has been directly related to the $\Delta_{3}$ [20]. The DFA technique was proposed in the context of time series analysis as a method to investigate the long-range correlation properties of nonlinear time series. In order to apply the DFA the time series is integrated and then divided into nonoverlaping $N / l$ boxes of equal length containing $l$ data points. Then a local trend is defined 
for each box, i.e. a linear fit to the integrated time series. Then the variance between the integrated time series and the local fit is computed for each box and averaged over all the boxes of size $l$. The average variance $F(l)$ depends on the box size. The analysis of this dependence allows to study the scaling properties of the system and the presence of long-range correlations. A linear relationship between $\log (F(l))$ and $\log (l)$ will indicate a scaling behavior. The slope $\alpha$ in the $\log (F(l))$ vs $\log (l)$ plot characterizes the scaling properties of the time series because it reflects a power law of the form $F(l) \sim l^{\alpha}$. In the case of a regular uncorrelated system the theoretical value of the slope is $\alpha=1 / 2$. In the case of GOE the expected value is $\alpha=1$ [20]. The DFA technique allows us to compare the $\delta_{n}$ fluctuation with the detrended one, $\delta_{n}^{\prime}$.

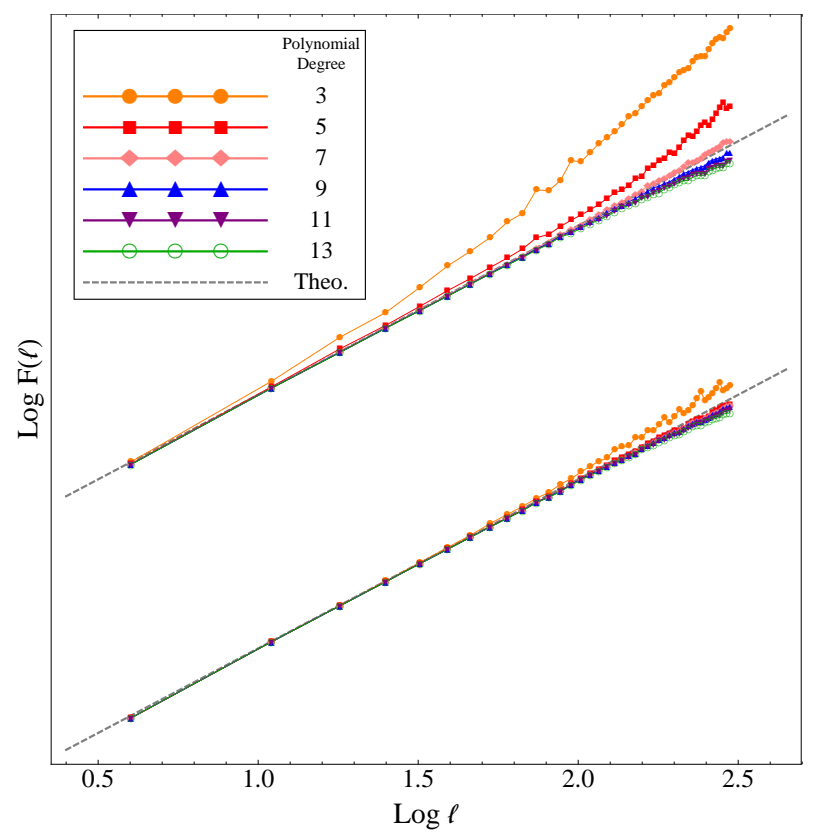

FIG. 5: Color online. Value of DFA variance $\log (F(l))$ averaged over the ensemble. The upper part shows $\log (F(l))$ computed using the DFA on the $\delta_{n}$, these plots have been displaced to avoid overlap. The lower part shows the $\log (F(l))$ from the DFA on the detrended $\delta_{n}^{\prime}$. Plots for different degrees of the polynomial fit used to unfold the quantum spectra are shown in both cases. The dashed line shows the theoretical result for the GOE.

A qualitative comparison on the DFA relation between $\log (F(l))$ and $\log (l)$ for the $\delta_{n}$ and $\delta_{n}^{\prime}$ fluctuations is shown on figure 5 . Once again, the improvement due to the detrended process is noticeable. The upper part shows $\log (F(l))$ as a function of $\log (l)$ obtained from a DFA calculation on the unfolded fluctuations $\delta_{n}$ for different degrees of the polynomial. The theoretical value for $\operatorname{GOE}(\alpha=1)$ is shown as a dashed gray line. It is evident how for low order polynomials the result diverges from the expected theoretical behavior when $l$ increases. Large values of $l$ correspond with long-range correlations on the time series. This plot shows how if the degree of the polynomial is too low the unfolding is capable of representing the correlations correctly, up to a certain range. On the other hand, if the polynomial degree is too big then the unfolding procedure destroys important long-range correlations. This effect is also observable on the upper part of figure 5 but is more subtle. The lower part of the same figure shows the result of the DFA on the detrended $\delta_{n}^{\prime}$ fluctuation. It is clear how the divergence observed for the low order polynomials has decreased. All the cases have a linear behavior which corresponds to the theoretical case. For very large values of $l$ the third order polynomial case begins to diverge, which means that some very long-range correlations are still missing. However, the range for which the DFA variance $\log (F(l))$ follows a linear relation has been substantially improved.

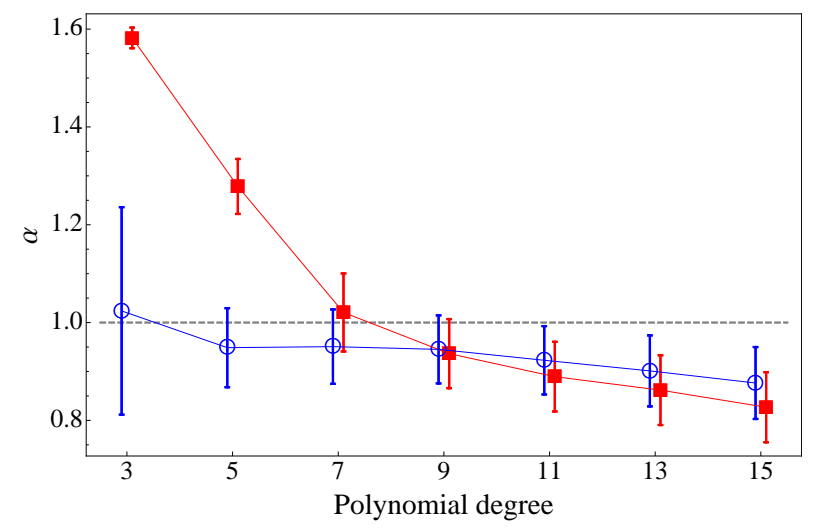

FIG. 6: Color online. Value of the $\alpha$ exponent of the power law behavior in the ensemble average calculation of the DFA for the fluctuation as a function of the degree of the polynomial fit used to unfold the quantum spectra. Two cases are shown, the $\alpha$ exponent values for the $\delta_{n}$ fluctuation (filled squares, red online) and the case for the $\delta_{n}^{\prime}$ fluctuation obtained detrending the previous one (empty circles, blue online). The dashed line shows the theoretical result for the GOE. The error bars show the standard deviation of the ensemble.

The results shown in figure 5 demonstrate qualitatively how detrending the fluctuation improves the range for which the long-range correlations of the system can be detected. However, the DFA allows us to observe this improvement in a quantitative way through the calculation of the value of the $\alpha$ exponent. Figure 6 compares the values obtained for $\alpha$ as a function of the order of the unfolding polynomial using the fluctuation $\delta_{n}$ (filled squares, red online) and the values obtained using the detrended fluctuation $\delta_{n}^{\prime}$ (empty circles, blue online). The theoretical behavior is shown as a gray dashed line. The improvement for small values of the polynomial 
degree is evident, after detrending the $\alpha$ exponent coincides with the predicted one. This improvement is observed up to the ninth order polynomial. For cases with larger values of the polynomial degree there is no improvement, again due to the effect mentioned before, i.e., a high order polynomial unfolding destroys important correlations which cannot be recovered detrending the fluctuations.

\section{CONCLUSIONS}

In order to study the statistical fluctuations of quantum spectra it is necessary to split the spectrum into two parts, a secular and a fluctuating one. This separation is done by means of an unfolding procedure. Several methodologies exist to unfold quantum spectra, but this procedure is not trivial. In this work we demonstrate that the usual measurements of quantum chaos can strongly depend on the unfolding procedure, i.e., an incorrect separation of the fluctuating part from the regular one can lead to erroneous conclusions about the chaoticity of a quantum system. In order to decrease this dependence we propose to remove the global trend which remains after an incorrect unfolding procedure. The detection of this global trend is the essential part of our proposal, which makes use of the Empirical Mode Decomposition techinique. Finally, we show that the detrending operation on the fluctuations removes to a large extent the dependence on the unfolding procedure for the usual measurements of the presence of quantum chaos. The effect of an incorrect unfolding is either to introduce spurious long-range correlations or to destroy important ones. In the first case the use of the EMD to detrend the fluctuations improves the range on which the correlations are correctly represented.

\section{ACKNOWLEDGMENTS}

This work was supported in part by grants from CONACyT, the Czech Science Foundation (202/09/0084) and the Czech Ministry of Education (MSM 0021620859).
[1] O. Bohigas, M.J. Giannoni and C. Schmit, Phys. Rev. Lett. 52, 1, (1984).

[2] M.V. Berry and M. Tabor, Proc. R. Soc. London, Ser. A 356, 375, (1977).

[3] H.J. Stöckmann, Quantum Chaos (Cambridge University Press, Cambridge, England, 1999).

[4] T.A. Brody, J. Flores, J.B. French, P.A. Mello, A. Pandey and S.S.M. Wong, Rev. Mod. Phys. 53, 385, (1981).

[5] J.M.G. Gómez, R.A. Molina, A. Relaño and J. Retamosa, Phys. Rev. E 66, 036209, (2002).

[6] K.F. Ratcliff Phys. Rev. C 3, 1, 117, (1971).

[7] J. Flores, M. Horoi, M. Müller and T. H. Seligman, Phys. Rev. E 63, 026204, (2001).

[8] J.B. French and S.S.M. Wong, Phys. Lett. 35B, 5, (1971).

[9] Henrik Bruus and Jean-Christian Anglès d'Auriac, Phys. Rev. B 55, 14, (1997).

[10] A. Relaño, J.M.G. Gómez, R.A. Molina, J, Retamosa and E. Faleiro, Phys. Rev. Lett. 89, 24, (2002).

[11] M.L. Mehta, Random Matrices (Academic, New York, 1991).

[12] Zhaohua Wu, Norden E. Huang, Steven R. Long and Chung-Kang Peng, PNAS, 104, 38, 14889, (September 18, 2007).

[13] Norden E. Huang, Zheng Shen, Steven R. Long, Manli
C. Wu, Hsing H Shih, Quanan Zheng, Nai-Chyuan Yen, Chi Chao Tung and Henry H. Liu, Proc, R. Soc. Lond. A, 454, 903, (1998).

[14] Norden E. Huang and Samuel S. P. Shen, Hilbert-Huang Transform and its applications (Interdisciplinary Mathematical Sciences - Vol. 5, 2005)

[15] Norden E. Huang, Man-Li C Wu, Steven R Long, Samuel S.P. Shen, Wendong Qu, Per Gloersen and Kuang L Fan, Proc. R. Soc. Lond. A, 459, 2317, (2003).

[16] E. Faleiro, J.M.G. Gómez, R.A. Molina, L. Muñoz, A. Relaño and J. Retamosa, Phys. Rev. Lett. 93, 244101, (2004).

[17] J.M.G. Gómez, A. Relaño, J. Retamosa, E. Faleiro, L. Salasnich, M. Vraničar and M.Robnik, Phys. Rev. Lett. 94, 084101, (2005).

[18] E. Landa, I. Morales, C. Hernández, J.C. López Vieyra and A. Frank, Rev. Mex. Fis. S 54 (3), 48, (2008).

[19] M.C. Gutzwiller, Jour. Math. Phys. 12, 343 (1971); M.C. Gutzwiller, Chaos in Classical and Quantum Mechanics (Springer, New York, 1990).

[20] M.S. Santhanam, Jayendra N. Bandyopadhyay and Dilip Angom, Phys. Rev. E 73, 015201(R), (2006).

[21] C.-K. Peng, S.V. Buldyrev, S. Havlin, M. Simons, H.E. Stanley and A.L. Goldberger, Phys. Rev. E 49, 2, (1994). 